\title{
Williamson fluid flow due to a nonlinearly stretching sheet with viscous dissipation and thermal radiation
}

\section{Ahmed M. Megahed (D)}

\section{Correspondence:}

ahmed.abdelbaqk@fsc.bu.edu.eg

Department of Mathematics,

Faculty of Science, Benha University, Benha, Egypt

\begin{abstract}
Williamson boundary layer fluid flow and heat transfer due to a nonlinearly stretching sheet is undertaken in this research. An important aspect of this study is that the thermal radiation and viscous dissipation phenomena are also included in this model. Because there is still much need for more accurate results, the viscosity and the fluid conductivity are assumed to vary with temperature. Our main purpose is to achieve a similar solution in this physical model. Here, we come across a highly nonlinear differential constitutive equations which are solved numerically after utilizing the shooting method. In this respect, it is noteworthy that the main characteristic developed here is that the study is made for the governing parameters and their effects on both the drag velocity and the heat transfer rate. Further, a significant effect of both radiation parameter and viscosity parameter on heating process is observed.
\end{abstract}

Keywords: Williamson fluid, Nonlinearly stretching sheet, Variable properties, Viscous dissipation

Mathematics subject classification: 76A05, 76D10, 65L10, 65L06

\section{Introduction}

During the last two decades, different immense attempts have been performed in fluid flow due to stretching sheet operation to give precious descriptions of standard manufacturing and industrial processes, such as polymer manufacturing, paper production, food preserving processes, crystal manufacturing, and petroleum filtering operation. For this sake and owing to these actual importance on this topic, many researchers have compelled to search about the novel findings which serve this field. The first contributions in this topic were inaugurated in discussion the fluid flow due to a stretched surface by Sakiadis [1]. The problem of fluid flow of Blasius type due to a stretching sheet, which has the application in the field of the drawing of plastic films has been studied by Crane [2]. Furthermore, we can resort to Chen and Char [3] to explore the mechanism of wall heatflux and its influence on an impermeable linearly stretching plate. Mohammadein and Gorla [4] investigated the heat transfer characteristics of a steady micropolar boundary layer fluid flow over a linearly stretching, continuous sheet. Shortly after the authors [4], Liu [5] perceived the general importance of hydromagnetic flow over a stretching sheet by applying it in a heat and mass transfer. Two years elapsed until Cortell [6] again presented steady flow and mass transfer approach to a second-grade fluid behavior based upon the

(c) The Author(s). 2019 Open Access This article is distributed under the terms of the Creative Commons Attribution 4.0 International License (http://creativecommons.org/licenses/by/4.0/), which permits unrestricted use, distribution, and reproduction in any medium, provided you give appropriate credit to the original author(s) and the source, provide a link to the Creative Commons license, and indicate if changes were made. 
concept of impermeable stretching sheet. In a later study, Chen [7] attempted to complete his research on some important mechanisms of MHD non-Newtonian power-law fluid over a stretching sheet. Recently, an increasing number of studies which concerning in exponentially stretching sheet can be introduced in Ref. [8-10] and in nonlinearly stretching sheet can be adequately described in Ref. [11-16].

In this work, we use the Williamson model, which was firstly studied since in 1929 by Williamson [17]. Due to the usefulness of this type of fluid, more of accurate researches are introduced in this topic [18-22]. In the framework of all previous studies and in an effort to analyze the Williamson fluid flow due to nonlinear stretching sheet with variable properties, thermal radiation, and viscous dissipation, this paper is introduced.

\section{Mathematical description for the physical model}

Currently, we will explain the physical problem under consideration by assuming that the moving fluid is a Williamson fluid with a time constant $\Gamma$. Williamson fluid embody the Cauchy stress tensor which fully governed by the extra stress tensor [18]. In addition, the motion for the fluid is yielded from the nonlinearly stretching sheet accompanying with the presence of the radiation and viscous dissipation phenomena. Also, the $x$-axis is selected along the sheet, while the $y$-axis is chosen in the orthogonal direction for the sheet (Fig 1). Due to the stretching process, this may be results in creating the velocity $U_{w}=c x^{m}$ for the fluid, where $c$ is a constant and $m$ is an exponent. Herein, we suppose that both the fluid thermal conductivity $\kappa$ and the fluid viscosity $\mu$ are altering with the temperature; however, the remaining fluid properties are constant.

After this former explanation and performing the approximations for the problem which we will study, we now have to present the mathematical equations which reflect what has been prescribed. These equations can be introduced in the following form [18]:

$$
\begin{aligned}
& \frac{\partial u}{\partial x}+\frac{\partial v}{\partial y}=0, \\
& u \frac{\partial u}{\partial x}+v \frac{\partial u}{\partial y}=\frac{1}{\rho_{\infty}} \frac{\partial}{\partial y}\left(\mu(T) \frac{\partial u}{\partial y}+\mu(T) \frac{\Gamma}{\sqrt{2}}\left(\frac{\partial u}{\partial y}\right)^{2}\right), \\
& u \frac{\partial T}{\partial x}+v \frac{\partial T}{\partial y}=\frac{1}{\rho_{\infty} c_{p}} \frac{\partial}{\partial y}\left(\kappa(T) \frac{\partial T}{\partial y}\right)+\frac{\mu(T)}{\rho_{\infty} c_{p}}\left(1+\frac{\Gamma}{\sqrt{2}} \frac{\partial u}{\partial y}\right)\left(\frac{\partial u}{\partial y}\right)^{2}-\frac{1}{\rho_{\infty} c_{p}} \frac{\partial q_{r}}{\partial y},
\end{aligned}
$$

we must refer that, the first equation represents the continuity equation while the second equation expresses the momentum, but the final equation reflects the energy equation. Always as usual, the characters $u$ and $v$ represent the components of the velocity vector in

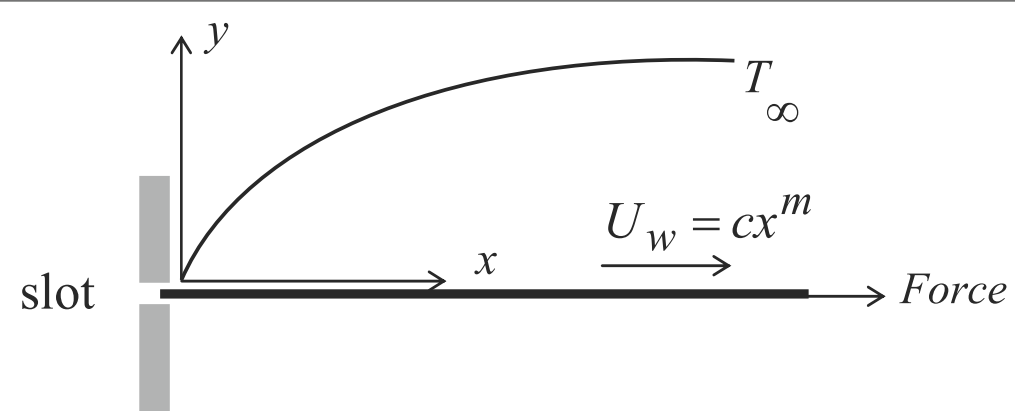

Fig. 1 Sketch of flow over nonlinearly stretching sheet 
the $x$ and $y$ directions, respectively. Also, the constant $\rho_{\infty}$ is called the fluid density at the ambient, the symbol $T$ refers to the temperature for the Williamson fluid, and the symbol $q_{r}$ describes the heat flux term which is yielded due to the radiation, while the constant property $c_{p}$ is the specific heat at constant pressure.

As reported previously, the Rosseland approximation [23], helped us to put $q_{r}$ as a function of temperature in the following form:

$$
q_{r}=-\frac{4 \sigma^{*}}{3 k^{*}} \frac{\partial T^{4}}{\partial y}
$$

where the constant $\sigma^{*}$ is the Stefan-Boltzmann and $k^{*}$ is the absorption coefficient. In this work, the coefficient $k^{*}$ must be coincide with the Rosseland approximation. As we observed from Eq. (4), the highly nonlinearity of the term $T^{4}$ can be simplified by using Taylor expansion about the constant value $T_{\infty}$ as $T^{4} \cong 4 T_{\infty}^{3} T-3 T_{\infty}^{4}$, after ignoring all higher-order terms [24].

In the same context, the nonlinear stretching process for the impermeable sheet and its temperature at the surface and away from it can be reflected in the following conditions:

$$
\begin{aligned}
& u=c x^{m}, \quad v=0, \quad T_{w}(x)=T_{\infty}+A x^{r} \quad \text { at } \quad y=0 \\
& u \rightarrow 0, \quad T \rightarrow T_{\infty}, \quad \text { as } \quad y \rightarrow \infty,
\end{aligned}
$$

where $T_{\infty}$ is the constant ambient temperature, $A, r$ are constants. Now, we will introduce the non-dimensional functions $f$ and $\theta$ in which they are function of the variable $\eta$ as follows:

$$
\eta=\left(\frac{c x^{m-1}}{v_{\infty}}\right)^{\frac{1}{2}} y, \quad \psi(x, y)=\left(c x^{m+1} v_{\infty}\right)^{\frac{1}{2}} f(\eta), \quad \theta(\eta)=\frac{T-T_{\infty}}{T_{w}-T_{\infty}},
$$

where $v_{\infty}$ is the kinematic viscosity at the ambient and $\psi(x, y)$ is the stream function which fulfill Eq. (1) according to:

$$
u=\frac{\partial \psi}{\partial y}, \quad v=-\frac{\partial \psi}{\partial x} .
$$

On the other hand, some of the important assumptions in this research is that the viscosity is changing exponentially with the temperature, while the thermal conductivity is altering linearly with temperature according to these equations [25]:

$$
\mu=\mu_{\infty} e^{-\alpha \theta}, \quad \kappa=\kappa_{\infty}(1+\varepsilon \theta) .
$$

The final relations present the coefficient $\mu_{\infty}$ and $\kappa_{\infty}$ which represent the viscosity and the thermal conductivity at the ambient, respectively. Also, from the same relations, the parameter $\alpha$ is the viscosity but the parameter $\varepsilon$ is the thermal conductivity.

Employing Eq. (7) in the principal Eqs. (1)-(3), we trusted that Eq. (1) is exactly satisfied, while the other equations reduce to:

$$
\begin{aligned}
& e^{-\alpha \theta}\left(\left(1+\delta f^{\prime \prime}\right) f^{\prime \prime \prime}-\alpha \theta^{\prime} f^{\prime \prime}\left(1+\frac{\delta}{2} f^{\prime \prime}\right)\right)+\left(\frac{m+1}{2}\right) f f^{\prime \prime}-m f^{\prime 2}=0, \\
& \frac{1}{\operatorname{Pr}}\left(\varepsilon \theta^{\prime 2}+(1+R+\varepsilon \theta) \theta^{\prime \prime}\right)+\left(\frac{m+1}{2}\right) f \theta^{\prime}-r f^{\prime} \theta+E c\left(1+\frac{\delta}{2} f^{\prime \prime}\right) f^{\prime \prime 2} e^{-\alpha \theta}=0,
\end{aligned}
$$


and the reduced boundary conditions are

$$
\begin{aligned}
& f(0)=0, \quad f^{\prime}(0)=1, \quad \theta(0)=1, \\
& f^{\prime} \rightarrow 0, \quad \theta \rightarrow 0, \quad \text { at } \quad \eta \rightarrow \infty,
\end{aligned}
$$

where $\delta=\left(\frac{\sqrt{2} c^{\frac{3}{2}} x^{\frac{3 m-1}{2}}}{\sqrt{v_{\infty}}}\right) \Gamma$ is the local Williamson fluid parameter, $\operatorname{Pr}=\frac{\mu_{\infty} c_{p}}{\kappa_{\infty}}$ is the Prandtl number, $R=\frac{16 \sigma^{*} T_{\infty}^{3}}{3 \kappa_{\infty} k^{*}}$ is the radiation parameter, and Ec $=\frac{U_{w}^{2}}{c_{p}\left(T_{w}-T_{\infty}\right)}=\frac{c^{2} x^{2 m-r}}{\mathrm{Ac}_{p}}$ is the local Eckert number. After finishing this analyzing, we observe that both the $\delta$ parameter and the Ec number are dependent of $x$. To overcome this dilemma which results in a non-similar solution for our problem, we should take $r=2 m=\frac{2}{3}$. So, these parameters take the form, $\delta=\left(\frac{\sqrt{2} c^{\frac{3}{2}}}{\sqrt{v_{\infty}}}\right) \Gamma$ is the Williamson fluid parameter and $\mathrm{Ec}=\frac{U_{w}^{2}}{c_{p}\left(T_{w}-T_{\infty}\right)}=\frac{c^{2}}{\mathrm{Ac} p}$ is the Eckert number.

\section{The local skin-friction coefficient and the local Nusselt number}

The physical description for our problem may be completed after analyzing the quantities of local skin-friction coefficient $C f_{x}$ and the local Nusselt number $N u_{x}$ as follows:

$$
\begin{aligned}
& C f_{x}=-2 \operatorname{Re}_{x}^{\frac{-1}{2}}\left(1+\frac{\delta}{2} f^{\prime \prime}(0)\right) f^{\prime \prime}(0) e^{-\alpha \theta(0)}, \\
& \mathrm{Nu}_{x}=-\operatorname{Re}_{x}^{\frac{1}{2}}(1+R+\varepsilon \theta(0)) \theta^{\prime}(0),
\end{aligned}
$$

$\operatorname{Re}_{x}=\frac{U_{w x}}{v_{\infty}}$ is the local Reynolds number. It is clear from Eq. (14) that the local skinfriction coefficient $C f_{x}$ is directly affected with both the viscosity parameter $\alpha$ and the Williamson fluid parameter $\delta$, while noted from Eq. (15) that the local Nusselt number is influenced by both the thermal conductivity parameter $\varepsilon$ and the radiation parameter $R$. Also, an implicit effects for another parameters on both the local Nusselt number and the local skin-friction coefficient can be occurred.

\section{Solution methodology}

It is impossible to get the exact solution for the system posed by (10)-(11), so we should resort to the numerical solution. Therefore, the numerical shooting method is adopted in this work to get the numerical solution of the proposed model. In this method, the governing Eqs. (10)-(11) along with the boundary conditions (12)-(13) were first reformulated as a system of first-order equations. This system cannot be solved with the infinite conditions which appear in (13). So, these conditions are replaced by appropriate finite guessing values for $f^{\prime \prime}(0)$ and $\theta^{\prime}(0)$ to start the solution. Whenever these values are appropriate, we hasten to get the solution. Also, the main objective of this section is to validate the numerical shooting solution to confirm the accuracy of our results. To perform this task, we must recall some previously published work which can be considered as a special case from our problem. We noticed that our problem can be reduced to the previously published work by Gorla and Sidawi [26] when $\delta=\alpha=\varepsilon=R=E c=r=0$ and $m=1$. Therefore, after performing the comparison which appear in Table 1, the shootingmethod proves to be accurate and very powerful. 
Table 1 Comparison of Nusselt number $\left(\mathrm{Nu}_{x} \mathrm{Re}_{x}^{\frac{-1}{2}}\right)$ for various values of $\operatorname{Pr}$ when $\delta=\alpha=\varepsilon=R=E C=$ $r=0$ and $m=1$

\begin{tabular}{lll}
\hline $\operatorname{Pr}$ & Gorla and Sidawi [26] & Present study \\
\hline 0.07 & 0.06562 & 0.065531 \\
0.20 & 0.16912 & 0.169117 \\
2.0 & 0.91142 & 0.911358 \\
7.0 & 1.89546 & 1.895453 \\
20.0 & 3.35391 & 3.353902 \\
\hline
\end{tabular}

\section{Results and discussion}

It must be affirmed that, this work can be completely self-contained, if we present all striking features for the non-Newtonian Williamson flow which yielded from a nonlinear stretching sheet after introducing the following graphs. The presented figures can recap all physical phenomena which can created from the introduced parameters in this section. So, the influence of the viscosity parameter $\alpha$ which ranging from 0.0 to 1.0 on both the velocity and temperature profiles are introduced in Figs. 2 and 3, respectively. As is foreseeable from these figures, the velocity distribution is altered with decreasing behavior by the presence of $\alpha$, while the temperature profiles has a reversible trend. Physically, the feature of Fig. 3 convince and enable us to predict the thermal thickness for large values of $\alpha$.

On the other hand, Figs. 4 and 5 study the effect of Williamson $\delta$ parameter on both the velocity and temperature profiles. These figures stipulated that the velocity distribution reached a maximum when we ignored the $\delta$ parameter, i.e., (for Newtonian fluid), but Fig. 4 reveals that the temperature distribution is slightly dependent on the manner in which the $\delta$ parameter varies. Further, Fig. 5 attains close agreement about the nonNewtonian Williamson fluids having large parameter $\delta$ which can be chosen to govern the rate of heat transfer insofar as it is possible.

The curves of the relationships between both $\varepsilon, R$ parameters and the temperature distribution are presented in Figs. 6 and 7, respectively. It is clear that a thermal curve of

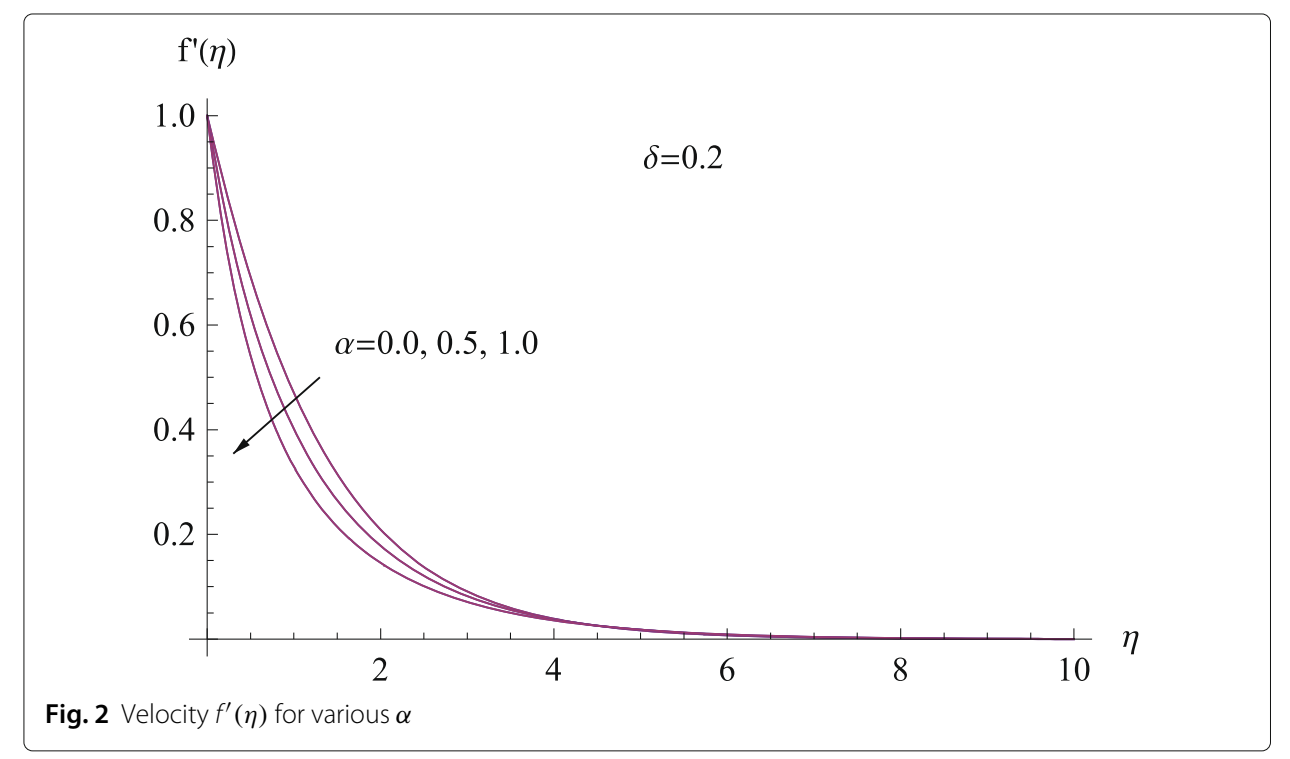




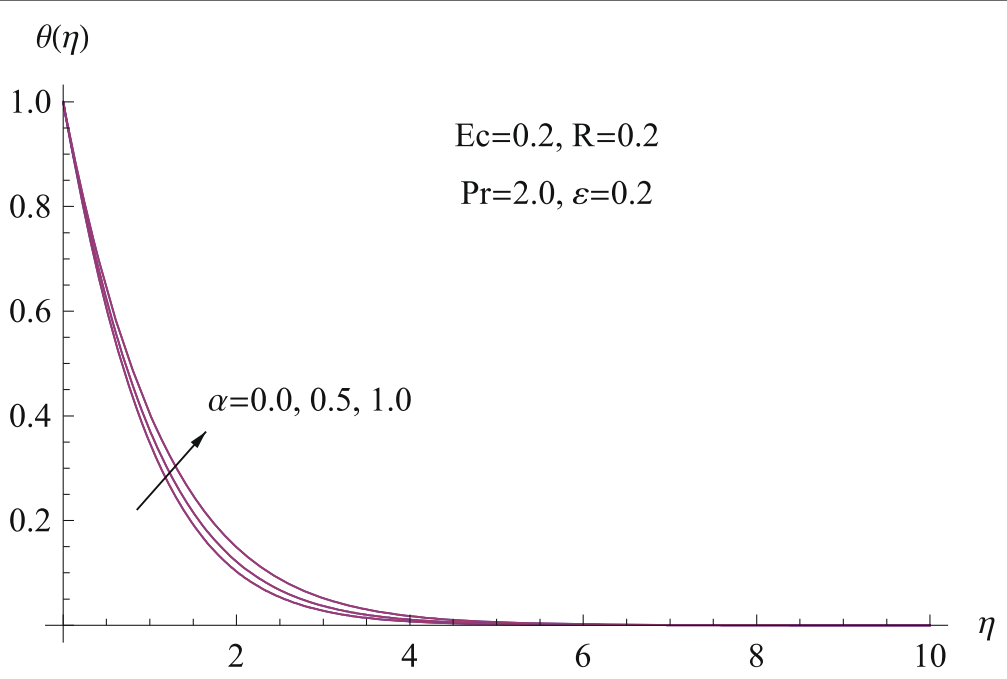

Fig. 3 Temperature $\theta(\eta)$ for various $\alpha$

the fluid which having less thermal conductivity was placed down the curve with more thermal conductivity, which means that a large $\varepsilon$ value induces more progress in the distribution of the temperature inside the thermal region. On the other hand, Fig. 6 indicates that the tested heat pattern through the parameter $\varepsilon$ was primarily affected by the Williamson fluids having variable conductivity. Further, thermal thickness and distribution of temperature with great radiation parameter $R$ are larger than those of distribution and thermal thickness with small radiation parameter $R$ as shown in Fig. 7.

In Fig. 8, a similar behavior for the Eckert number Ec will be given, which elucidates the response of the thermal region for the viscous dissipation phenomena. It is important to bear in mind that the presence of viscous dissipation phenomena has the probability of inducing the growth of both the temperature distribution and the thickness of the thermal layer. Physically, when viscous dissipation is considered in the model, the mechanism of convective heat is strengthen and this results in thickening the thermal layer.

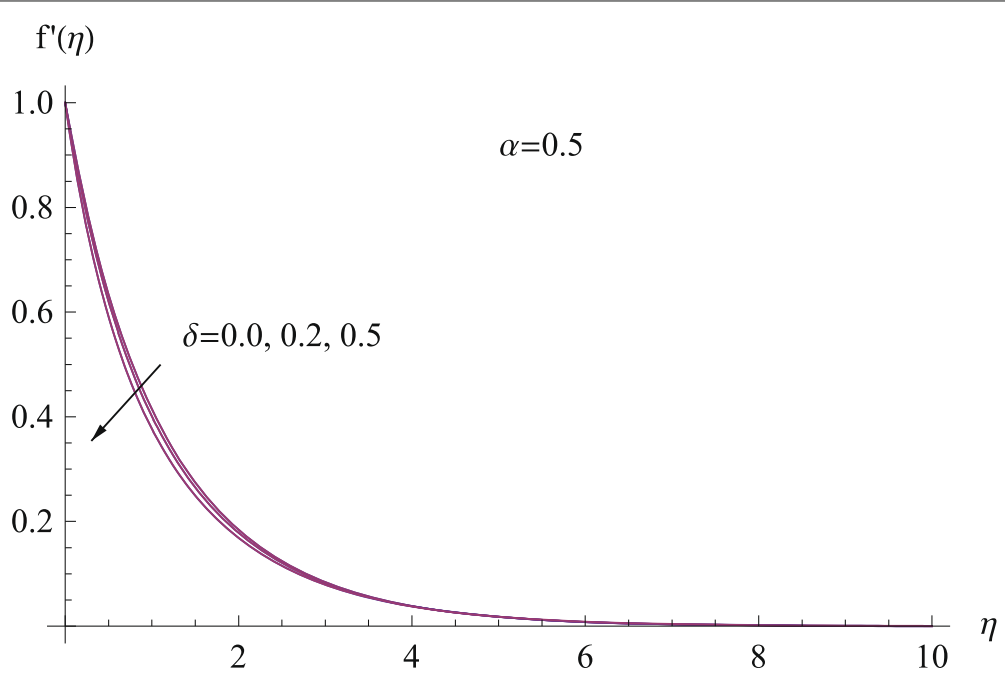

Fig. 4 Velocity $f^{\prime}(\eta)$ for various $\delta$ 


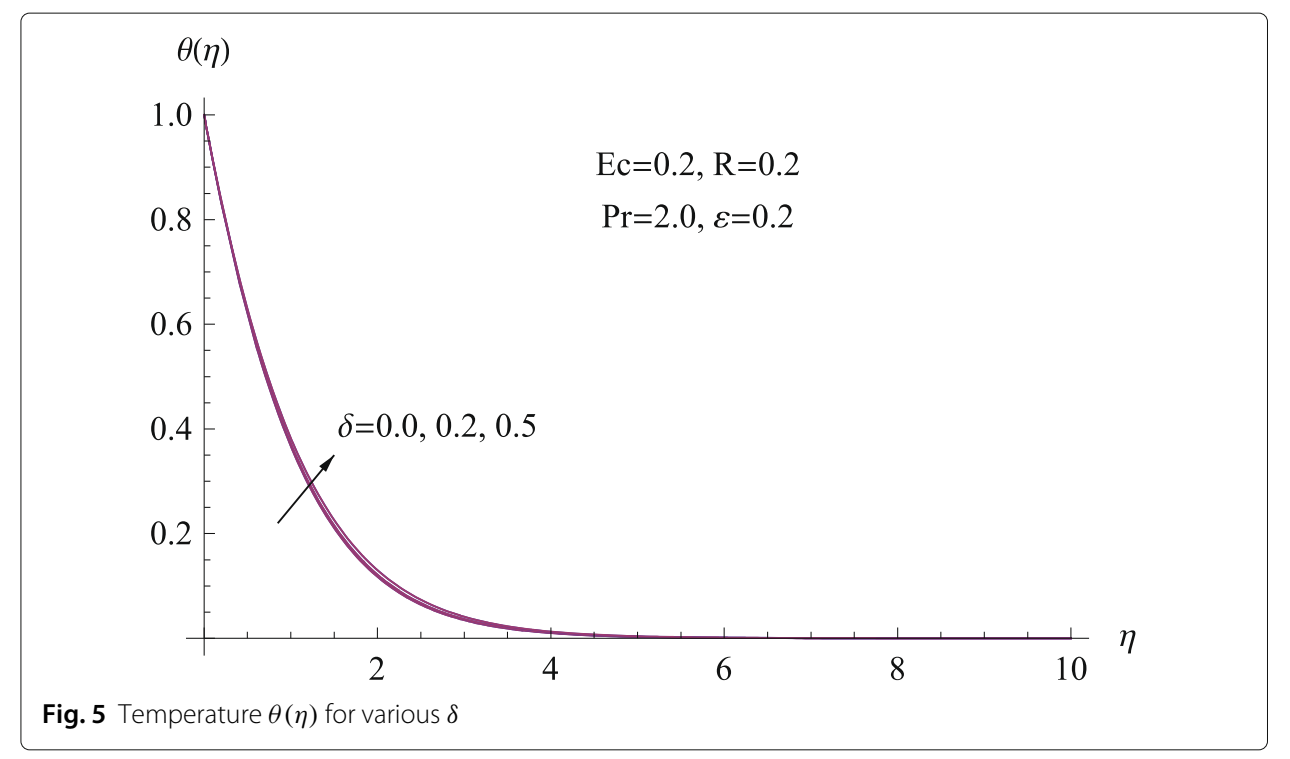

For returning to the work at hand and seeking to measure the impact of all physical parameters governing the Williamson fluid flow on both the local skin-friction coefficient $\frac{1}{2} \operatorname{Re}_{x}^{\frac{1}{2}} \mathrm{Cf}_{x}$ and the local Nusselt number $\mathrm{Re}_{x}^{\frac{-1}{2}} \mathrm{Nu}_{x}$, one needs to calculate the data which appear in Table 2. Of particular interest, introduced here are the Eckert number $E c$, the viscosity parameter $\alpha$, and the non-Newtonian Williamson parameter $\delta$, in which they result in a diminishing behavior for both the local skin-friction coefficient and the local Nusselt number. On the other hand, an augmentation behavior in the local Nusselt number is noted from this study for increasing both the thermal conductivity parameter $\varepsilon$ and the thermal radiation parameter $R$, but a reduction trend was for the local skin-friction coefficient.

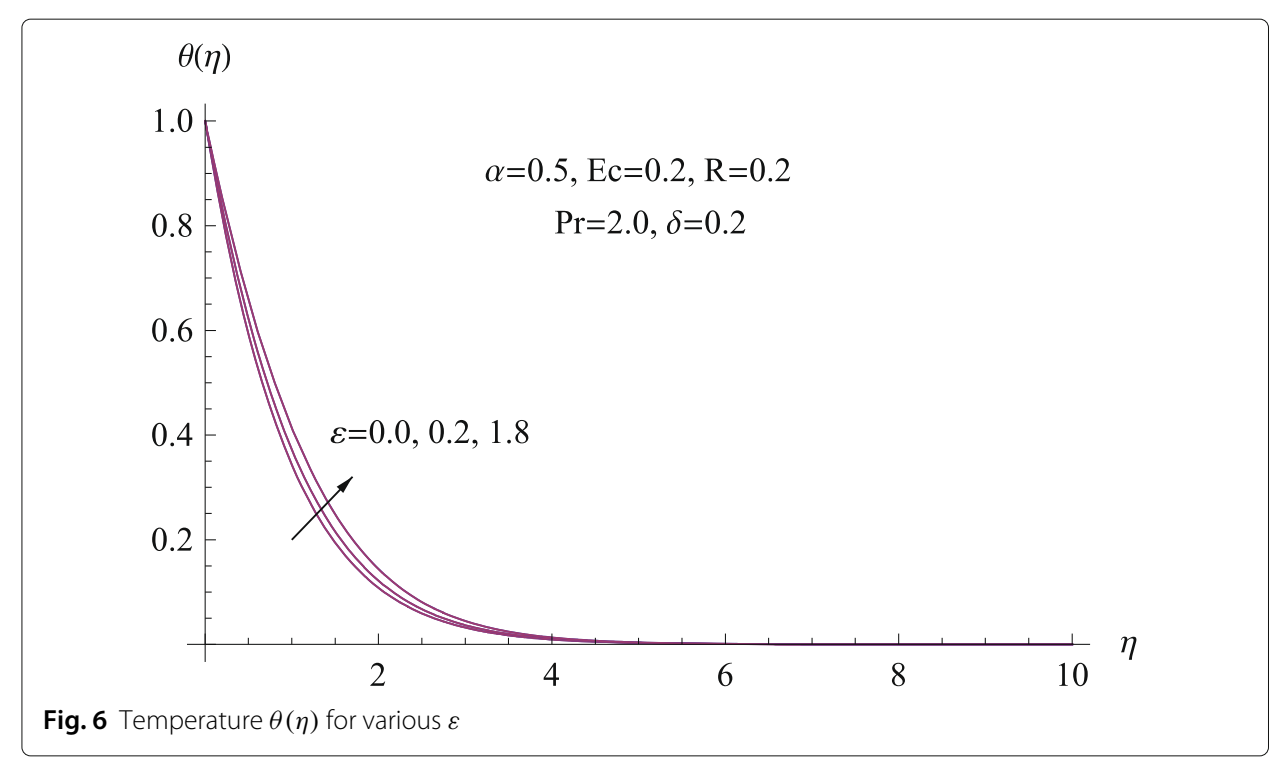




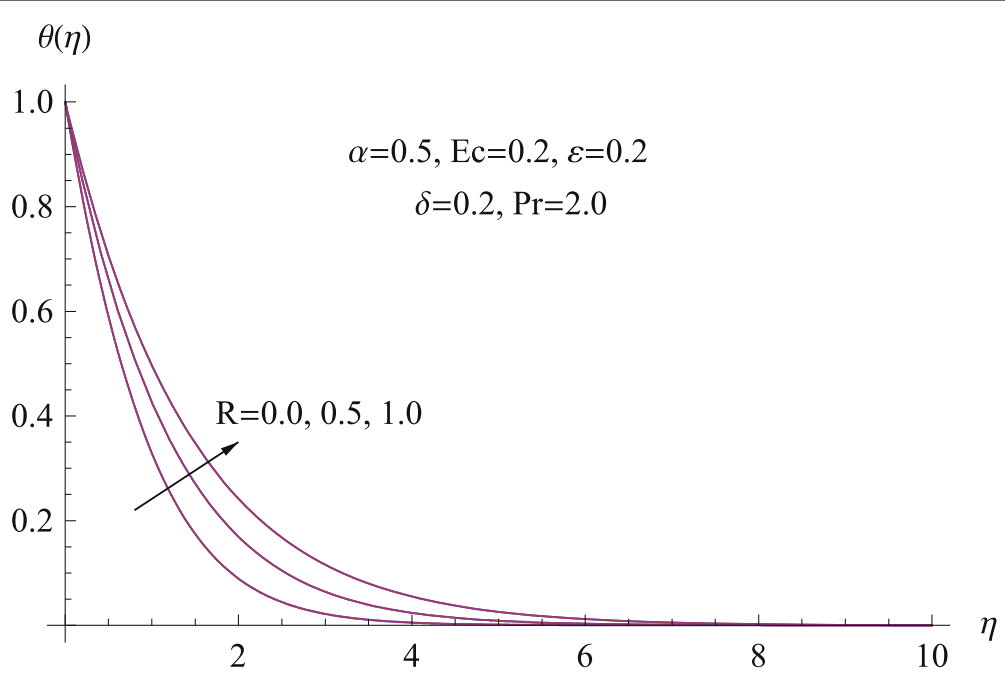

Fig. 7 7. Temperature $\theta(\eta)$ for various $R$

\section{Concluding remarks}

According to the yielded analysis for the proposed Williamson fluid flow, some of main interesting results which are drawn from this study are established below in an elaborated form as follows: (i) Both the thermal radiation parameter and the Eckert number have the influence to enhance the temperature distribution, thicken the thermal region, thus increase the local Nusselt number and decrease the local skin-friction coefficient. (ii) Increasing both the viscosity parameter and the Williamson parameter will result in a rise in the temperature distribution, and hence a diminishing behavior for both the rate of heat transfer and the local skin-friction coefficient. (iii) The thermal conductivity parameter has an impact in enhancing the temperature distribution, hence an increasing behavior for the local Nusselt number.

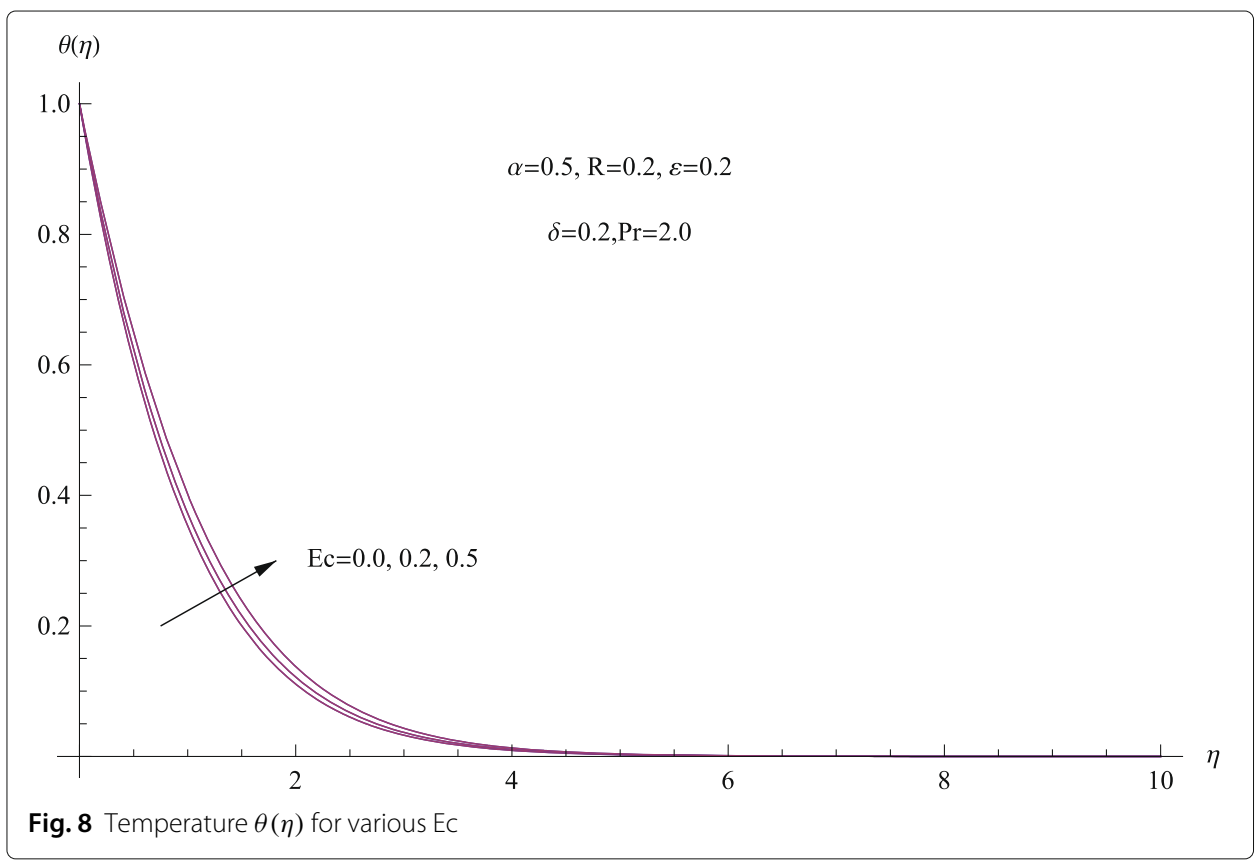


Table 2 Values of $\frac{1}{2} \operatorname{Re}_{x}^{\frac{1}{2}} C f_{x}$ and $\operatorname{Re}_{x}^{\frac{-1}{2}} N u_{x}$ for various values of $\alpha, \delta, \varepsilon, R$, and Ec with $m=\frac{1}{3}, r=\frac{2}{3}, \operatorname{Pr}=2.0$

\begin{tabular}{lllllll}
\hline$\alpha$ & $\delta$ & $\varepsilon$ & $R$ & $\mathrm{EC}$ & $\frac{1}{2} \operatorname{Re}_{x}^{\frac{1}{2}} \mathrm{Cf}_{x}$ & $\operatorname{Re}_{x}^{\frac{-1}{2}} \mathrm{Nu}_{x}$ \\
\hline 0.0 & 0.2 & 0.2 & 0.2 & 0.2 & 0.659037 & 1.33148 \\
0.5 & 0.2 & 0.2 & 0.2 & 0.2 & 0.546574 & 1.27392 \\
1.0 & 0.2 & 0.2 & 0.2 & 0.2 & 0.439826 & 1.20470 \\
0.5 & 0.0 & 0.2 & 0.2 & 0.2 & 0.567823 & 1.28576 \\
0.5 & 0.2 & 0.2 & 0.2 & 0.2 & 0.546574 & 1.27392 \\
0.5 & 0.5 & 0.2 & 0.2 & 0.2 & 0.506503 & 1.24864 \\
0.5 & 0.2 & 0.0 & 0.2 & 0.2 & 0.549231 & 1.21855 \\
0.5 & 0.2 & 0.2 & 0.2 & 0.2 & 0.546574 & 1.27392 \\
0.5 & 0.2 & 0.5 & 0.2 & 0.2 & 0.543210 & 1.34926 \\
0.5 & 0.2 & 0.2 & 0.0 & 0.2 & 0.549629 & 1.19309 \\
0.5 & 0.2 & 0.2 & 0.5 & 0.2 & 0.542856 & 1.37819 \\
0.5 & 0.2 & 0.2 & 1.0 & 0.2 & 0.538196 & 1.51929 \\
0.5 & 0.2 & 0.2 & 0.2 & 0.0 & 0.548239 & 1.36147 \\
0.5 & 0.2 & 0.2 & 0.2 & 0.2 & 0.546574 & 1.27392 \\
0.5 & 0.2 & 0.2 & 0.2 & 0.5 & 0.544103 & 1.14333 \\
\hline
\end{tabular}

\section{Acknowledgements}

The helpful criticism of honorable editor and the anonymous reviewers in the preparation of this paper is gratefully acknowledged.

\section{Funding}

Not applicable.

\section{Availability of data and materials}

Not applicable.

\section{Author's contributions}

The author read and approved the final version of the manuscript.

\section{Competing interests}

The author declares that he has no competing interests.

\section{Publisher's Note}

Springer Nature remains neutral with regard to jurisdictional claims in published maps and institutional affiliations.

Received: 3 August 2018 Accepted: 5 November 2018

Published online: 07 June 2019

\section{References}

1. Sakiadis, B. C.: Boundary layer behaviour on continuous moving solid surfaces. I. Boundary layer equations for two-dimensional and axisymmetric flow. II. Boundary layer on a continuous flat surface. III. Boundary layer on a continuous cylindrical surface. AlChE J. 7, 26-28 (1961)

2. Crane, L. J., Angew, Z.: Flow past a stretching plate. Math. Phys. 21, 645-647 (1970)

3. Chen, C. K., Char, M. I.: Heat transfer of a continuous stretching surface with suction or blowing. J. Math. Anal. Appl. 135, 568-580 (1988)

4. Mohammadein, A. A., Gorla, R. S. R.: Heat transfer in a micropolar fluid over a stretching sheet with viscous dissipation and internal heat generation. Int. J. Numer. Meth. Heat Fluid Flow. 11, 50-58 (2001)

5. Liu, I. C.: A note on heat and mass transfer for a hydromagnetic flow over a stretching sheet. Int. Commun. Heat Mass Transf. 32, 1075-1084 (2005)

6. Cortell, R.: MHD flow and mass transfer of an electrically conducting fluid of second grade in a porous medium over a stretching sheet with chemically reactive species. Chem. Eng. Process. 46, 721-728 (2007)

7. Chen, C.: Magneto-hydrodynamic mixed convection of a power-law fluid past a stretching surface in the presence of thermal radiation and internal heat generation/absorption. Int. J. Non-Linear Mech. 44, 596-603 (2009)

8. Rehman, F. U., Nadeem, S., Haq, R. U.: Heat transfer analysis for three-dimensional stagnation-point flow over an exponentially stretching surface. Chinese J. Phys. 55, 1552-1560 (2017)

9. Rehman, F. U., Nadeem, S.: Heat transfer tnalysis for three-dimensional stagnation-point flow of water-based nanofluid over an exponentially stretching surface. J Heat Transf. 140(5), 0524011-0524017 (2018)

10. Hayat, T., Nadeem, S.: Flow of 3D Eyring-Powell fluid by utilizing Cattaneo-Christov heat flux model and chemical processes over an exponentially stretching surface. Results Phys. 8, 397-403 (2018) 
11. Megahed, A. M.: Variable viscosity and slip velocity effects on the flow and heat transfer of a power-law fluid over a non-linearly stretching surface with heat flux and thermal radiation. Rheol. Acta. 51, 841-874 (2012)

12. Mahmoud, M. A. A., Megahed, A. M.: Non-uniform heat generation effects on heat transfer of a non-Newtonian fluid over a non-linearly stretching sheet. Meccanica. 47, 1131-1139 (2012)

13. Megahed, A. M.: Flow and heat transfer of a non-Newtonian power-law fluid over a non-linearly stretching vertical surface with heat flux and thermal radiation. Meccanica. 50, 1693-1700 (2015)

14. Saif, R. S., Hayat, T., Ellahi, R., Muhammad, T., Alsaedi, A.: Stagnation-point flow of second grade nanofluid towards a nonlinear stretching surface with variable thickness. Results Phys. 7, 2821-2830 (2017)

15. Hayat, T., Sajjad, R., Muhammad, T., Alsaedi, A., Ellahi, R.: On MHD nonlinear stretching flow of Powell-Eyring nanomaterial. Results Phys. 7, 535-543 (2017)

16. Megahed, A. M.: Flow and heat transfer of non-Newtonian Sisko fluid past a nonlinearly stretching sheet with heat generation and viscous dissipation. J. Braz. Soc. Mech. Sci. Eng. 40, 492 (2018). https://doi.org/doi.org/10.1007/ s40430-018-1410-3

17. Williamson, W. W.: The flow of pseudoplastic materials. Ind. Eng. Chem. 21, 1108-1111 (1929)

18. Nadeem, S., Hussain, S. T., Lee, C.: Flow of a Williamson fluid over a stretching sheet. Braz. J. Chem. Eng. 30, 619-625 (2013)

19. Khan, N. A., Khan, H. A.: A Boundary layer flows of non-Newtonian Williamson fluid. Nonlinear Eng. 3, 107-115 (2014)

20. Malik, M. Y., Salahuddin, T.: Numerical solution of MHD stagnation point flow of Williamson fluid model over a stretching cylinder. Int. J. Nonlin. Sci. Num. 16, 161-164 (2015)

21. Malik, M. Y., Bibi, M., Khan, F., Salahuddin, T.: Numerical solution of Williamson fluid flow past a stretching cylinder and heat transfer with variable thermal conductivity and heat generation/absorption. AIP Adv. 6, 035101 (2016)

22. Vittal, C., Reddy, M. C. K., Vijayalaxmi, T.: MHD stagnation point flow and heat transfer of Williamson fluid over exponential stretching sheet embedded in a thermally stratified medium. Glob. J. Pur. Appl. Math. 13, 2033-2056 (2017)

23. Raptis, A.: Flow of a micropolar fluid past a continuously moving plate by the presence of radiation. Int. J. Heat Mass Tran. 41, 2865-2866 (1998)

24. Raptis, A.: Radiation and viscoelastic flow. Int. Commun. Heat Mass Tran. 26, 889-895 (1999)

25. Mahmoud, M. A. A., Megahed, A. M.: MHD flow and heat transfer in a non-Newtonian liquid film over an unsteady stretching sheet with variable fluid properties. Can. J. Phys. 87, 1065-1071 (2009)

26. Gorla, R. S. R., Sidawi, I.: Free convection on a vertical stretching surface with suction and blowing. Appl. Sci. Res. $\mathbf{5 2}$ 247-257 (1994)

\section{Submit your manuscript to a SpringerOpen ${ }^{\odot}$ journal and benefit from:}

- Convenient online submission

- Rigorous peer review

- Open access: articles freely available online

- High visibility within the field

- Retaining the copyright to your article

Submit your next manuscript at $\gg$ springeropen.com 